\section{Salmonella typhimurium epidural empyema in an HIV infected patient}

\author{
Wissem Hachfi,' Foued Bellazreg,' \\ Mohamed Ladib, ${ }^{2}$ Naoufel Kaabia, ${ }^{1}$ \\ Mabrouk Khalifa, ${ }^{1}$ Hedi Krifa, ${ }^{2}$ \\ Amel Letaief'
}

'Department of Infectious Diseases, and Internal Medicine, Farhat Hached University Hospital; ${ }^{2}$ Department of Neurosurgery, Sahloul University Hospital, Sousse, Tunisia

\section{Abstract}

Salmonella focal intracranial infections are reported rarely. They tend to occur in immunocompromised patients. We present here a case of Salmonella typhimurium epidural empyema, with osteomyelitis of the adjacent frontal bone, in a 37-year-old human immunodeficiency virus positive man who presented with a three-day history of headache, fever, and sweats. He was treated successfully with antibiotics and surgical drainage.

\section{Introduction}

Non-typhoidal salmonella (NTS) infection is a common occurrence among human immunodeficiency virus (HIV) infected patients, and salmonella bacteremia is a frequent finding. The estimated incidence of salmonellosis among patients with acquired immunodeficiency syndrome (AIDS) has been 20- to 100fold more than that among the general population. ${ }^{1}$ Before the era of AIDS, central nervous system (CNS) localization of NTS infection was reported rarely and meningitis was the most common location, described more frequently in children., ${ }^{2,3}$ Salmonella focal intracranial infections are unusual in HIV infected patients. We report a case of an HIV patient with a Salmonella typhimurium infection presenting as epidural empyema, with adjacent osteomyelitis of the frontal bone.

\section{Case Report}

A 37-year-old HIV positive man presented at our department on December 10, 2007 with a three-day history of headache, fever, and sweats. Physical examination revealed a Glasgow coma score of $15 / 15$, a temperature of $38.2^{\circ} \mathrm{C}$, a blood pressure of $110 / 70 \mathrm{~mm} \mathrm{Hg}$, a pulse rate of 90 beats/min, and a respiratory rate of 20 breaths/min. No neurological deficit was detected. HIV infection was diagnosed 10 years ago, and antiretroviral therapy (zidovudine-didanosine-lopinavir/ritonavir) had been started in October 2007, when he had esophageal candidiasis with a CD4 cell count of $12 / \mathrm{mm}^{3}(3 \%)$ and an HIV viral load of 110,000 RNA copies/mL.

The laboratory results showed low hemoglobin $(11.1 \mathrm{~g} / \mathrm{dL})$, normal WBC count $\left(6100 / \mathrm{mm}^{3}\right.$ with 4500 neutrophils), and normal platelets $\left(349,000 / \mathrm{mm}^{3}\right)$. The prothrombin time was normal (75\%), as were the values of glucose, creatinine, and electrolytes. Absolute CD4 cell count was $71 / \mathrm{mm}^{3}(9 \%)$. HIV viral load was not performed. On lumbar puncture the cerebrospinal fluid was normal. The chest X-ray was unremarkable. A contrasted computed tomography scan (CT) (Figure 1) and magnetic resonance imaging (MRI) (Figure 2) of the head revealed epidural empyema in the left frontal region, associated with adjacent osteolytic lesions in the frontal bone. There were no signs of brain herniation.

The patient was treated initially with par-
Correspondence: Wissem Hachfi, Unit of Infectious Diseases, Farhat Hached Hospital, 4000 Sousse, Tunisia. E-mail: hachfi_wissem@hotmail.com

Key words: Salmonella typhimurium, epidural empyema, human immunodeficiency virus.

Conflict of interest: the authors report no conflict of interests.

Received for publication: 6 August 2009. Revision received: 1 November 2009.

Accepted for publication: 4 November 2009.

This work is licensed under a Creative Commons Attribution 3.0 License (by-nc 3.0).

(C) Copyright W. Hachfi et al., 2009

Licensee PAGEPress, Italy

Infectious Disease Reports 2009; 1:e5

doi:10.4081/idr.2009.e5

enteral cefotaxime (12 $\mathrm{g}$ daily) combined with metronidazole (1500 mg daily). Antiretroviral therapy was continued. After 48 hours of antibiotic therapy, fever resolved but the
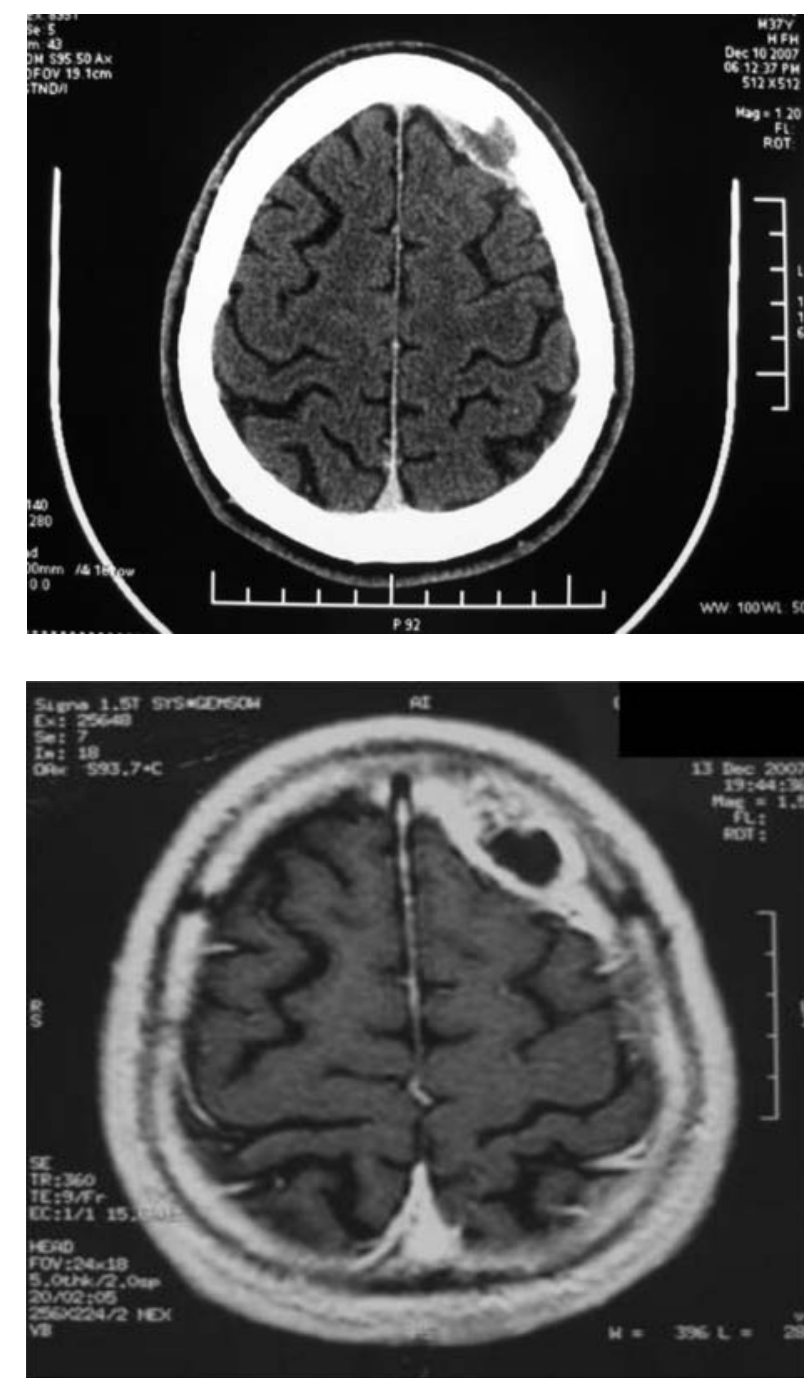

Figure 1. Computed tomography scan of the head showing a $4 \times 1.5-\mathrm{cm}$ left epidural lentiform hypodensity with an adjacent frontal bone defect.
Figure 2. Enhanced T1-weighted magnetic resonance image showing a 5-cm epidural collection with adjacent osteolytic lesions in the left frontal area. 
patient complained of persistant headache. A craniotomy was performed on December 14, producing epidural purulent fluid that was drained. Pus culture yielded a pure growth of a gram-negative bacillus, which was identified as Salmonella typhimurium. A disk diffusion susceptibility test showed the isolate to be susceptible to cefotaxime, ciprofloxacin, and choramphenicol, but resistant to amoxicillin and cotrimoxazole. The antibiotic therapy was changed to ciprofloxacin per os (1000 mg daily). Within two days following the drainage of the fluid collection, the headache subsided. Blood cultures showed no growth after seven days of incubation. Stool culture was not performed. The patient was discharged without any neurological complications. Ciprofloxacin was continued for a period of 12 weeks. A repeat CT scan of the head performed at the end of antibiotic therapy showed the resolution of the epidural empyema (Figure 3). At nine months of follow-up, the patient remained clinically very well. Viral load was of $<25$ RNA copies/mL and CD4 cell count was $198 / \mathrm{mm}^{3}$ (12\%).

\section{Discussion}

The incidence of NTS infection among patients with AIDS exceeds that among the general population. ${ }^{1}$ Frequently salmonellosis in this population is complicated by bacteremia, especially in patients with CD4 counts of $<100 / \mathrm{mm}^{3}{ }^{4}$ However, suppurative complications, particularly those affecting the CNS, have been reported rarely. ${ }^{1}$ NTS meningitis is the most common location of CNS salmonella infection, but remains rare. ${ }^{5}$ Either brain abscess, subdural empyema, or epidural empyema mostly occurs as a complication of salmonella meningitis, but may be an isolated infection.${ }^{6,7}$ Our patient had epidural empyema without meningitis. $S$. enteritidis and $S$. typhimurium are the most frequently encountered serotypes associated with salmonella CNS infections. ${ }^{8}$

NTS are food-borne pathogens that primarily cause gastroenteritis, then can reach the bloodstream via the lymphatics, especially in immunocompromised persons. ${ }^{8}$ However, not all individuals with invasive NTS infections have concurrent diarrhea or isolation of the bacteria from the stool. ${ }^{9}$ In a Spanish study, only $34 \%$ of AIDS patients with NTS bacteremia had diarrhea, and salmonella were isolated from the stools in only $4 \%{ }^{9}$ Sarria $e t$ $a l$., in their study of 11 cases of $S$. enteritidis brain abscesses, found antecedent gastroenteritis in only four patients. ${ }^{6}$ Neither intestinal infection nor salmonella bacteremia had been detected in our patient. However, the possibility of transient hematogenous spread of $S$.

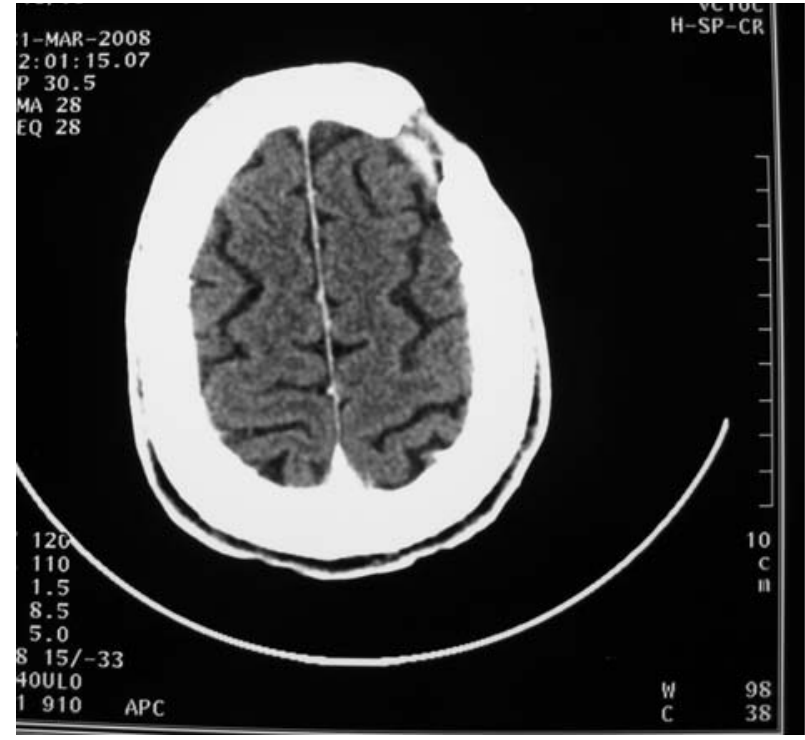

Figure 3. Computed tomography scan of the head performed at the end of antibiotic therapy showing the removal of the epidural empyema.

typhimurium from a pre-existing asymptomatic intestinal infection could not be eliminated.

Although NTS localized infection has not been reported in the literature as a possible manifestation of immune restitution syndrome, we think this supposition could be advanced for our patient. In fact we observed this infection two months after the beginning of antiretroviral therapy, concomitant with an increase of CD4 count. However, our hypothesis lacked the HIV viral load decrease parameter, which was not available when this NTS infection was diagnosed.

Clinical symptoms associated with epidural empyema are nonspecific: headache, fever, seizures, decreased level of consciousness, focal neurological deficit. In our patient, these symptoms were limited to headache and fever. Consequently the diagnosis often is unsuspected or delayed. Therefore early imaging studies, namely CT scan and MRI of the head, are required to make a prompt diagnosis. ${ }^{6}$

Our patient had skull osteomyelitis complicating the epidural empyema. Salmonella skull osteomylitis is extremely rare. It has been noted in two patients with salmonella brain abscess before the era of AIDS..$^{10,11}$ More recently Mastroianni et al. reported a case of frontal salmonella osteomyelitis without intracranial complications in an HIV patient, ${ }^{12}$ and Aliaga et al. reported a case of salmonella subdural and epidural cerebral empyema with concomitant osteomyelitis of the frontal bone in an HIV patient. ${ }^{13}$ In addition salmonella osteomyelitis associated with epidural infection was described in patients with sickle cell disease. . $^{14,15}$

High mortality rates have been reported for patients with intracranial infections caused by NTS, and a significant number of survivors developed permanent neurological sequelae., ${ }^{7,16}$
Treatment failure was observed particularly when antibiotics were used alone without surgery. ${ }^{15}$ The use of an adequate antimicrobial regimen combined with early surgical drainage will result in the best probability of cure. ${ }^{7,17}$ The most reliable choices appear to be third-generation cephalosporins and fluoroquinolones because of their high activity against most salmonella strains with high brain tissue and CSF concentrations. Either chloramphenicol or cotrimoxazole could be used as an alternative drug if there is a contraindication to cephalosporins and quinolones. ${ }^{16}$ The recommended duration of treatment is at least four weeks in meningitis and at least six weeks in brain abscess or empyema. ${ }^{16,17}$ Our patient received prompt antimicrobial therapy and surgical drainage, and the duration of antibiotic therapy was prolonged to 12 weeks because he had skull osteomyelitis. He was cured without any sequelae and no relapse was noted at nine months of follow-up.

\section{Conclusions}

Salmonella focal intracranial infections are rare but should be considered by clinicians as a cause of acute CNS disorder in patients with advanced HIV disease. Early diagnosis and treatment are important in order to improve the prognosis of this CNS infection.

\section{References}

1. Sperber SJ, Schleupner CJ. Salmonellosis during infection with human immunodeficiency virus. Rev Infect Dis 1987;9:925-34. 
2. Rodriguez RE, Valero V, Watanakunakorn C. Salmonella focal intracranial infections: review of the world literature (1884-1984) and report of an unusual case. Rev Infect Dis 1986;8:31-41.

3. Cohen A, Freundlich E, Greif Z. Nontyphoid salmonella septicemia. Harefuah 1987;1112:5-7.

4. Pegues DA, Ohl ME, Miller SI. Salmonella species, including Salmonella typhi. In: Mandell GL, Bennett JE, Dolin R, editors. Principles and practice of infectious diseases. 6th edn. Philadelphia: Elsevier, 2005, pp 2636-50.

5. Leonard MK, Murrow JR, Jurado R, et al. Salmonella meningitis in adults infected with HIV: case report and review of the literature. Am J Med Sci 2002;323:266-8.

6. Sarria JC, Vidal AM, Kimbrough RC. Salmonella enteritidis brain abscess: case report and review. Clin Neurol Neurosurg 2000;102:236-9.

7. Kuruvath S, Basu S, Elwitagala JP, et al.
Salmonella enteritidis brain abscess in sickle cell disease patient: case report and review of the literature. Int $\mathrm{J}$ Infect Dis 2008;12:298-302.

8. Hart CA, Beeching NJ, Duerden BI. Infections in AIDS. J Med Microbiol 2000; 49:947-67.

9. Casado JL, Valdezate S, Calderon NE, et al. Zidovudine therapy protects against Salmonella bacteremia recurrence in human immunodeficiency virus-infected patients. J Infect Dis 1999;179:1553-6.

10. Mage J, Martin P. Abcès epidural par ostéomyélite, unique manifestation d'une infection à paratyphique B. Acta Neurol Psychiatr Belg 1958;58:861-7.

11. Suzuki Y, Sugiyama Y, Ishii R, et al. Brain abscess caused by Salmonella typhi: case report. J Neurosurg 1976;45:709-11.

12. Mastroianni CM, Vullo V, Delia S. Cranial Salmonella abscess with parietal bone osteomyelitis in an HIV-infected patient. AIDS 1992;6:749-50.
13. Aliaga L, Mediavilla JD, López de la Osa A, et al. Nontyphoidal salmonella intracranial infections in HIV-Infected Patients. Clin Infect Dis 1997;25:1118-20.

14. Martino AM, Winfield JA. Salmonella osteomyelitis with epidural abscess. A case report with review of osteomyelitis in children with sickle cell anemia. Pediatr Neurosurg 1990;16:321-5.

15. Diebold P, Humbert J, Djientcheu Vde P, et al. Salmonella epidural abscess in sickle cell disease: failure of the nonsurgical treatment. J Natl Med Assoc 2003;95:10958.

16. Owusu-Ofori A, Scheld WM. Treatment of Salmonella meningitis: two case reports and review of the literature. Int $\mathrm{J}$ Infect Dis 2003;7:53-60.

17. Infection in Neurosurgery. The rational use of antibiotics in the treatment of brain abscess. Working Party of the British Society for Antimicrobial Chemotherapy. Br J Neurosurg 2000;14:525-30. 